\title{
Optimal protection of stabilised dry live bacteria from bile toxicity in oral dosage forms by bile acid adsorbent resins
}

Article

Accepted Version

Manuscript

Edwards, A. D., Chatterjee, P., Mahbubani, K. T., Reis, C. M. and Slater, N. K.H. (2010) Optimal protection of stabilised dry live bacteria from bile toxicity in oral dosage forms by bile acid adsorbent resins. Chemical Engineering Science, 65 (16). pp. 4844-4854. ISSN 0009-2509 doi:

https://doi.org/10.1016/j.ces.2010.05.030 Available at https://centaur.reading.ac.uk/7999/

It is advisable to refer to the publisher's version if you intend to cite from the work. See Guidance on citing.

To link to this article DOI: http://dx.doi.org/10.1016/j.ces.2010.05.030

Publisher: Elsevier

All outputs in CentAUR are protected by Intellectual Property Rights law, including copyright law. Copyright and IPR is retained by the creators or other copyright holders. Terms and conditions for use of this material are defined in the End User Agreement. 


\section{CentAUR}

Central Archive at the University of Reading

Reading's research outputs online 


\title{
Optimal Protection of Stabilised Dry Live Bacteria from Bile Toxicity in Oral Dosage Forms by
}

Bile Acid Adsorbent Resins

\author{
Alexander D. Edwards a, c \\ Pratichi Chatterjee \\ Krishnaa T. Mahbubani \\ Cassilda M. Reis \\ Nigel K. H. Slater ${ }^{b}$ \\ Department of Chemical Engineering and Biotechnology, University of Cambridge \\ New Museums Site, Pembroke Street, Cambridge, CB2 3RA, UK \\ ${ }^{a}$ Phone: <44> 1223763969 \\ Fax: <44> 1223334796 \\ Email: ade24@cam.ac.uk, a.d.edwards@reading.ac.uk \\ ${ }^{\mathrm{b}}$ Phone: <44> 1223762953 \\ Fax: <44> 1223334796 \\ Email: nkhs2@cam.ac.uk \\ ${ }^{c}$ Current address: Reading School of Pharmacy \\ Whiteknights, PO Box 224, Reading RG6 6AD
}

\begin{abstract}
We previously found that dried live bacteria of a vaccine strain can be temporarily sensitive to bile acids and suggested that Bile Adsorbing Resins (BAR) can be used in oral vaccine tablets to protect dried bacteria from intestinal bile. Here, we report a quantitative analysis of the ability of BAR to exclude the dye bromophenol blue from penetrating into matrix tablets and also sections of hard capsule shells. Based on this quantitative analysis, we made a fully optimised formulation, comprising $25 \% \mathrm{w} / \mathrm{w}$ of cholestyramine in V caps ${ }^{\mathrm{TM}}$ HPMC capsules. This gave effectively $100 \%$ protection of viability from $4 \%$ bile, with 4200 -fold more live bacteria recovered from this formulation compared to unprotected dry bacteria. From the image analysis, we found that the filler material or compaction force used had no measurable effect on dye exclusion but did affect the rate of tablet hydration. Increasing the mass fraction of BAR gave more exclusion of dye up to $25 \% \mathrm{w} / \mathrm{w}$, after which a plateau was reached and no further dye exclusion was seen. More effective dye exclusion was seen with smaller particle sizes (i.e. cholestyramine) and when the BAR was thoroughly dried and disaggregated. Similar results were found when imaging dye penetration into capsule sections or tablets. The predictions of the dye penetration study were tested using capsules filled with dried attenuated Salmonella vaccine plus different BAR types, and the expected protection from bile was found, validating the imaging study. Surprisingly, depending on the capsule shell material, some protection was given by the capsule alone without adding BAR, with Vcaps ${ }^{\mathrm{TM}}$ HPMC capsules providing up to 174 -fold protection against $1 \%$ bile; faster releasing Vcaps Plus ${ }^{\mathrm{TM}}$ HPMC capsules and Coni Snap ${ }^{\mathrm{TM}}$ gelatin capsules gave less protection.
\end{abstract}

Keywords: Adsorption, Bacteria, Bile, Biological and Biomolecular Engineering, Dissolution, Formulation, Vaccine. 


\section{Introduction}

Therapeutic live bacteria are administered orally in vaccines against enteric pathogens (Levine 2006), for re-colonisation of commensal flora and for delivery of therapeutic molecules (Prakash and Jones 2005). Live bacterial vaccines are attractive because they are administered orally, they can be stabilised for room temperature distribution (Bullifent et al. 2000), and they induce robust immune responses. In order to generate this robust immune response live bacteria must be delivered to the upper small intestine since oral administration of killed bacteria gives a weaker immune response (Kantele et al. 1991). However, the human gastrointestinal tract is highly effective at killing bacteria ingested with food. Therefore, live bacterial vaccines must be formulated in a way that protects viability not only during storage but also during oral delivery. We discovered that after drying in a room temperature stable form, bacteria of a live vaccine strain become highly sensitive to acid and bile (Edwards and Slater 2008, 2009). Stomach acid toxicity can be bypassed using enteric coatings, but after release into the intestine, dry bacteria are then exposed to bile which contains high concentrations of bile acids that are toxic to many bacteria (Begley et al. 2005). Furthermore, the concentration of bile encountered will depend on the exact location of release from an enteric coated dosage and on the time of administration relative to a meal (Begley et al. 2005). Therefore, for reliable delivery of a fixed dose of live cells, dried live bacteria must be protected from bile.

Previously, two approaches have been proposed to protect live bacteria from bile. A variety of microencapsulation techniques have been developed that can protect live bacteria from acid and/or bile (Prakash and Jones 2005). These microencapsulation techniques have two drawbacks for vaccine delivery; firstly they do not release the bacteria in the gut and so the function of the live vaccine is compromised, and secondly they are costly to manufacture. Alternatively, some bacteria can be grown in conditions that increase their inherent bile resistance, for example pre-conditioning by adding bile (Begley et al. 2005) or other detergents (Kimoto et al. 2002) to culture medium. However, this effect is strain dependent and may be modulated by subsequent lyophilisation.

We have developed a new formulation for protecting dried bacteria from bile that relies on adsorption of toxic bile acids in the outer part of an oral dosage form (Edwards and Slater 2009). We suggested using Bile acid Adsorbing Resins $\left(\mathrm{BAR}^{1}\right)$ to adsorb bile acids, which are safe, orally administered ion-exchange resins that have found previous clinical use to block intestinal bile acid reabsorption and lower cholesterol levels (Bergen et al. 1959). Initial studies with a model attenuated Salmonella vaccine strain showed that 250 -fold more live bacteria were recovered in a $1 \%$ bile solution when the BAR cholestyramine was added at $33 \% \mathrm{w} / \mathrm{w}$ to compressed matrix tablets, compared to tablets made with filler alone (Edwards and Slater 2009). Although this tablet formulation gave significant bile protection, there was still a 2.5-fold decrease in live bacteria recovery when comparing tablets dispersed in bile vs buffer alone, representing a $60 \%$ loss. This suggested a potential to optimise the formulation and achieve higher protection levels.

In the current study, we used an imaging system to make quantitative measurements of dye penetration into tablets and capsules containing BAR. We found that compaction force, filler and BAR composition influence tablet hydration rates, swelling, and dye penetration, and these results suggest an optimal composition for maximal bile protection. We then confirmed the predictions of the imaging analysis using live bacteria, and showed that capsules could be made which completely protected room temperature stable dried bacteria of a vaccine strain against high concentrations of bile.

\section{Methods}

\subsection{Reagents}

Bromophenol blue, LB agar, ampicillin, kanamycin, M9 salts and all growth medium supplements, ox bile (purified dried preparation), pig bile, the anion-exchange resins cholestyramine, 400 mesh Dowex 1X2, 50 mesh Dowex 1X2, triethylaminoethyl (TEAE) cellulose, diethylaminoethyl (DEAE) cellulose, QAE sephadex (triethylaminoethyl functionalised, A-50 grade), 400 mesh

\footnotetext{
${ }^{1}$ Abbreviation: BAR: Bile Adsorbing Resin
} 
triethylamino propyl functionalised silica gel (TEAP silica), chitosan preparations A (powder, 'low molecular weight' type, $92 \%$ deacetylated, viscosity $35 \mathrm{cPs}$ at $1 \%$ in acetic acid) and B (flakes, 'middle viscosity' type, viscosity 337 at $1 \%$ in acetic acid) and the fillers microcrystalline cellulose (MCC, Avicel PH101), starch and calcium phosphate were purchased from Sigma-Aldrich (Poole, Dorset, UK). Details of the anion exchange resins used are provided in table 1. Lactose filler was Tablettose 80 (Meggle, Wasserburg, Germany). Sylgard 184 polydimethylsiloxane (PDMS) was polymerised in shallow dishes according to the manufacturer's instructions (Dow Chemical Company, Staines, UK). Phosphate buffer used for dissolution was $0.025 \mathrm{M}$ potassium dihydrogen phosphate and $0.025 \mathrm{M}$ disodium hydrogen phosphate at $\mathrm{pH}$ 7.0. Bile solutions were made by dissolving ox or pig bile in phosphate dissolution buffer followed by 0.2 micron filtration.

Samples of Vcaps ${ }^{\mathrm{TM}}$ HPMC capsules, Vcaps Plus ${ }^{\mathrm{TM}}$ HPMC capsules, and Coni Snap ${ }^{\mathrm{TM}}$ gelatin capsules were a kind gift from Capsugel (Bornem, Belgium); some of the same capsules were also supplied by Value Healthcare Company (Sheffield UK).

\subsection{Imaging dye penetration into tablets and capsule sections}

To make tablets, BAR and fillers were thoroughly mixed in carefully weighed proportions as indicated. In all cases proportions of BAR are indicated as a mass fraction (i.e. \% w/w). 50-100mg samples of the mixed powders were then pressed in a $7 \mathrm{~mm}$ die in a modified $\mathrm{KBr}$ press (Port-A-Press from International Crystal Laboratories, supplied by Crystan Ltd., Poole) a torque of 8N.m was used unless otherwise indicated (applied with a Roebuck torque wrench) to make compressed matrix tablets $1-2 \mathrm{~mm}$ thick.

The apparatus used to image tablet dissolution is illustrated in figure 1a. Tablets were sandwiched between two $2 \mathrm{~mm}$ thick sheets of polydimethylsiloxane (PDMS), supported by two clear acrylic plates, with sufficient force to ensure good contact between the PDMS and the tablet surfaces, resulting in the dye solution only contacting the edges of the tablet and not the top or bottom surface. An O-ring was used to form a dissolution chamber around the tablet, and springs were used to support the top acrylic plate to allow accurate positioning and ensure the tablet was not compressed, and they remained at a fixed distance apart during dissolution. A $1.5 \mathrm{mM}$ solution of bromophenol blue in 100 $\mathrm{mM}$ phosphate buffer $\mathrm{pH} 7.0$ was added to each tablet using a syringe through an injection port fitted in the acrylic plate and hole in the PDMS sheet. Transmitted light was provided by a Medalight light box (Morco, Nottingham, UK) and the entire apparatus was placed within an opaque box to ensure only transmitted light was imaged. Images were captured onto VHS videocassettes using a JVC TKC1381EG CCD camera mounted on a dissecting microscope. Videos were transcribed to digital format using Roxio Easy VHS to DVD (www.Roxio.com/store) and subsequently analysed using ImageJ software (Abramoff et al. 2004). Image size was calibrated relative to the initial tablet diameter for each video analysed.

To image capsule dissolution, $2 \mathrm{~mm}$ thick rings were cut from the middle portion of size 00 Vcaps $^{\mathrm{TM}}$ HPMC capsules and placed on a PDMS membrane. The indicated mixtures of BAR and filler were added into the ring with a funnel and the filled capsule ring was then sandwiched between PDMS sheets with sufficient force to ensure no leakage at the edges of the capsule segment. Bromophenol blue solution was added and imaging was conducted as above for tablets.

\subsection{Analysis of dye diffusion and tablet hydration}

Videos were analysed to determine the extent of dye and water penetration; examples of these images are shown in figure 2a. The transmitted light illuminated tablet appears grey, due to light scattering by the particulate nature of the tablet. As the tablet hydrates during dissolution in buffer alone, light scattering is reduced, resulting in a bright white ring appearing. Dye penetration, in contrast, results in a blue colour. Therefore, when BAR is present and binds to dye during hydration, three distinct areas can be defined: a central dark grey area representing dry tablet; around this, a bright white area, representing hydrated tablet without dye; and finally a blue area, representing dye penetration. As well as seeing water and dye penetration, tablets containing powders such as cholestyramine swell on hydrating, resulting in expansion of the tablet. At each time three fronts were identified and measured (Fig. 1b): a swelling front, a dye exclusion front, and a water penetration front; these were named $\mathrm{r}_{\text {swell }}$, $r_{\text {exclusion }}$ and $r_{\text {water }}$. After the tablet was fully hydrated, a stable endpoint was observed and the endpoint 
$r_{\text {swell }}$ and $r_{\text {exclusion }}$ were measured (Fig. 1c). When no BAR was present in the tablet, the dye penetrated at the same rate as water and no clear zone was seen, with the whole tablet becoming blue.

\subsection{Particle size analysis}

Particle sizing was performed on a Morphologi G3 (Malvern Instruments, Malvern, UK). Small samples of wet and dried 400 mesh Dowex 1X2, cholestyramine and TEAP silica were analysed using the automated sample dispersion unit to disperse the powders on a glass slide according to the manufacturer's instructions, followed by image acquisition and analysis. The average circular equivalent (CE) diameter of the particles for a volume distribution of each sample was taken after filtering to identify single particles. Aggregated particles were also identified and the ratio of aggregated to nonaggregated particles calculated.

\subsection{Live bacterial vaccine capsule testing}

In the data presented in Figure 6, the mouse plague vaccine strain SL3261/pAH34L was used (Bullifent et al. 2000); similar results were obtained using the antibiotic-free plasmid maintenance strain SLDAPD/pUC18I (Edwards and Slater 2008, 2009; Garmory et al. 2005). Bacteria were counted using LB agar plates containing either ampicillin for SLDAPD/ pUC18I or kanamycin for SL3261/pAH34L to ensure only vaccine bacteria were counted. Bacteria were grown and dried as described in (Bullifent et al. 2000; Edwards and Slater 2008, 2009), and then several replicate dried preparations were pooled and ground to a homogeneous powder in a mortar and mixed with dry MCC to make a free-flowing powder for formulation and filling into capsules. This powder typically contained $2-5 \times 10^{6} \mathrm{cfu} / \mathrm{mg}$ and was stored in a vacuum desiccator at room temperature until testing; the preparation showed less than $10 \%$ loss of viability over a 2 month period. Powdered dried bacteria were thoroughly mixed with the indicated proportions of fillers and BAR; between 20-30mg bacteria were added per $1 \mathrm{~g}$ total powder, to ensure that $6-10 \mathrm{mg}$ powdered dried bacteria were included per capsule. The mixed powder was manually filled into size 00 capsules of the indicated shell material using a Cap-M-Quick (Value Healthcare Company, Sheffield UK); a $6.4 \mathrm{~mm}$ diameter steel ball bearing was added to the capsule to sink it during dissolution. The capsules were tested for bile resistance as follows: tablets were weighed and placed individually in $50 \mathrm{ml}$ test tubes, to which $25 \mathrm{ml}$ portions of phosphate buffer $\pm 1 \%$ or $4 \%$ ox bile at $37{ }^{\circ} \mathrm{C}$ were added followed by incubation for 50 minutes at $37^{\circ} \mathrm{C}$; similar counts were seen at a $1.5 \mathrm{~h}$ timepoint indicating no further loss of cells after this time (data not shown). Samples were taken after thorough mixing, serially diluted and plated onto LB agar plates with the appropriate antibiotic, followed by overnight incubation. The resulting colonies were counted to quantify CFU/ml, and then expressed in terms of the original dried bacteria weight, i.e. in CFU/mg of dried bacteria.

\section{Results}

Extensive research has been published using imaging to study the hydration and swelling of delayed release matrix tablets containing hydroxypropyl methylcellulose HPMC (Bettini et al. 1994; Chirico et al. 2007). However, little is known about the penetration of molecules into tablets during hydration and dissolution, and we decided to study this process using an imaging system. In order to simplify the study, a 2-dimensional system was chosen where tablets were sandwiched between clear plates, previously used to study gel formation and erosion during dissolution of HPMC tablets (Bettini et al. 1994; Chirico et al. 2007). Bile acids do not absorb visible or ultraviolet light, nor are they fluorescent; standard measurement methodologies include enzymatic assays, HPLC and mass spectroscopy. For these reasons, it is not possible to measure bile acid distribution in real time. Therefore anionic dye bromophenol blue was used instead, because it absorbs visible light strongly and binds to anion-exchange resins in a manner similar to bile acids. Preliminary experiments illustrated that BAR could block penetration of this dye in a highly simplified system (Edwards and Slater 2009).

\subsection{Effect of compaction force and filler on tablet hydration}

When analysing images of water and dye penetration into BAR tablets, we identified three parameters that vary with time during dissolution $r_{\text {swell }}, r_{\text {exclusion }}$ and $r_{\text {water }}$ (Fig. 1b), and also measured the endpoint $r_{\text {swell }}$ and $r_{\text {exclusion }}$ once the tablet was fully hydrated and $r_{\text {water }}$ had reached 0 (Fig. 1c). Our initial analysis focussed on deciding which of these parameters are likely to give the best indication of bile acid exclusion during tablet hydration. 
In initial experiments with a mixture of 50\% cholestyramine and 50\% MCC we found high tablet-to-tablet variation in the rate of dissolution of tablets. We suspected this was caused by variable compaction force provided by the manual press used to make tablets, resulting in tablets with variable porosity; increased compaction force decreases tablet porosity resulting in slower hydration (Yoshihashi et al. 1998). To confirm that varying compaction force alters hydration kinetics, we tested tablets made with differing torques. Images of two examples illustrate the slower hydration seen with tablets pressed at $30 \mathrm{~N} . \mathrm{m}$ vs 8 N.m torque (Fig. 2a). The slower kinetics can be seen quantitatively when the three parameters were measured at different time points and plotted against time to illustrate the kinetics of dissolution (Fig. 2b, c). We determined the time taken for complete hydration (i.e. when $r_{\text {water }}$ reaches 0 ) for multiple replicate tablets, and these times increased reproducibly with increasing compaction force (Fig. 2d). A similar relationship between torque and hydration speed was seen for tablets made with $50 \%$ cholestyramine and 50\% starch (Fig. 2e). Changing filler had a dramatic effect on hydration kinetics; at all torques, starch filler tablets had longer hydration times than MCC filler tablets (Fig. 2d, e). The high standard deviations in hydration times reflect the high tablet-to-tablet variation described (Fig. 2d, e).

\subsection{Influence of filler and BAR type on dye penetration into tablets}

In spite of the effect on hydration kinetics seen by varying compaction force and filler type, the depth of penetration of dye into tablets appeared to be highly reproducible; for example, the images in figure $2 \mathrm{a}$ appear remarkably similar except for the differing timepoints. Confirming this, the endpoint $r_{\text {exclusion }}$ was unaffected by varying compaction force or filler and therefore independent of hydration kinetics (Fig. 2f,g). Three further fillers with varying particle size, solubility and swelling characteristics were compared using a fixed torque of $8 \mathrm{~N} . \mathrm{m}$, and very similar dye exclusion was seen with $50 \% \mathrm{w} / \mathrm{w}$ cholestyramine mixed with each of the fillers tested (Fig. 3a). In contrast, substituting polystyrene sulphonate for the BAR, a resin with similar polystyrene-DVB powder support, but with a negative sulphonate ligand instead of positive quaternary amines which does not bind bromophenol blue and cannot block dye entry, resulted in complete penetration of dye and a $r_{\text {exclusion }}$ of 0 (Fig. 3a).

To determine the effect of BAR type on dye exclusion, a range of different anion-exchange resins were compared, mixed at $50 \%$ with MCC. Cholestyramine is a powdered form of the polystyrene/DVB resin Dowex 1X2 which is available in a variety of particle sizes (table 1).

Intriguingly, although 400-mesh Dowex 1X2 gave similar dye exclusion to cholestyramine, the 50-mesh Dowex 1X2 showed no dye exclusion with an endpoint $r_{\text {exclusion }}$ of 0 (Fig. 3b). QAE Sephadex, with the same functional groups as cholestyramine but a crosslinked dextran backbone, showed similar endpoint $r_{\text {exclusion }}$ and $r_{\text {swell }}$ to cholestyramine. In contrast, TEAE and DEAE cellulose showed very little $r_{\text {swell }}$, and had $r_{\text {exclusion }}$ very close to the $r_{\text {swell }}$, indicating excellent anion exclusion. TEAP silica showed a similar pattern to the cellulose-backbone BARs.

As well as these synthetic anion-exchange resins, two different preparations of the naturallyderived polycationic polymer chitosan were tested, one of low molecular weight (Chitosan A) and one of medium molecular weight (Chitosan B), both resulted in a low endpoint $r_{\text {swell }}$, but only Chitosan A gave dye exclusion (Fig. 3b). This may to be due to the particle size: Chitosan A was a fine powder, whereas Chitosan B was large flakes, or may be related to their different molecular weights.

\subsection{Effect of BAR proportion and type on dye exclusion in tablets}

Varying the proportion of BAR had a significant effect on dye exclusion. For the BAR cholestyramine, both $\mathrm{r}_{\text {exclusion }}$ and $\mathrm{r}_{\text {swell }}$ decreased as the proportion of BAR was reduced below 25\%, however, even as little as $1 \%$ resulted in a small but significant clear zone (Fig. 4a). No major increase in either $r_{\text {exclusion }}$ or $r_{\text {swell }}$ was seen above $25 \%$, indeed tablets made of $100 \%$ cholestyramine displayed a similar dye exclusion pattern to tablets containing 50\% cholestyramine (data not shown), and 50\% cholestyramine gave marginally lower $r_{\text {swell }}$ and $r_{\text {exclusion }}$ than $25 \%$ (Fig. 4a). This indicated that the optimal concentration of cholestyramine in compressed matrix tablets for maximal dye exclusion is $25 \%$.

By varying the proportion of BAR it was possible to quantitatively compare three forms of Dowex 1X2 which have the same support and ionic group but differing particle sizes (table 1). Although cholestyramine and 400 mesh Dowex 1X2 gave similar effects at 50\% (Fig. 3b), when reduced to lower proportions these BAR behaved very differently. Cholestyramine powder at $25 \%$ gave maximal $r_{\text {swell }}$ 
and $\mathrm{r}_{\text {exclusion at }}$ of $8 \mathrm{~mm}$ and $5.8 \mathrm{~mm}$ respectively, in contrast 400 mesh Dowex $1 \mathrm{X} 2$ only gave $6.2 \mathrm{~mm}$ and $3 \mathrm{~mm}$ respectively (Fig. 4b). As the proportion of 400 mesh Dowex 1X2 was reduced to 10\% and below, effectively no clear excluded zone was seen. In contrast, as little as $1 \%$ cholestyramine gave a detectable $\mathrm{r}_{\text {exclusion}}$, and $2.5 \%$ cholestyramine gave similar exclusion to $25 \% 400$ mesh Dowex 1X2 suggesting that this BAR is quantitatively inferior to cholestyramine. Even larger particles give less protection, with 50 mesh Dowex 1X2 giving no exclusion at 25 or $50 \%$ (Fig. 4d).

When handling 400 mesh Dowex 1X2, it became clear it was supplied partially hydrated and aggregated. To investigate the effect of hydration, samples of cholestyramine and 400 mesh Dowex 1X2 were dried in a vacuum desiccator, and weighed to determine water loss. Although cholestyramine showed less than 5\% weight loss over 1 week, the 400 mesh Dowex 1X2 had a weight loss of 37.7\% over 17 days, resulting in a reduction in mean particle size from a CE of $58 \mu \mathrm{m}$ to $50 \mu \mathrm{m}$. Strikingly, after drying, this sample became a free-flowing powder that could be easily mixed with filler. The effect of water on aggregation was clearly seen during particle size analysis; $26 \%$ of the wet preparation comprised aggregated particles, in contrast only $12 \%$ of particles were aggregated in the dried sample. The importance of BAR hydration and aggregation was seen when the dried preparation was tested in the imaging system. At 25 and 50\%, little difference was seen in endpoint $r_{\text {exclusion }}$ between wet and dry preparations. However, at 1 and $10 \%$ a significant increase in $r_{\text {exclusion }}$ was seen with the dried sample (Fig. 4b,c).

TEAP silica has a similar particle size to 400 mesh Dowex $1 \mathrm{X} 2(\mathrm{CE}=61 \mu \mathrm{m})$ but contrasts by having rigid particles that do not swell on hydration. TEAP silica was dry and free-flowing with no aggregation detectable during particle size analysis. Although TEAP silica has larger particle size and does not swell, when compared over a range of proportions a similar pattern of $r_{\text {exclusion }}$ was observed to cholestyramine. As with cholestyramine, similar $r_{\text {exclusion }}$ was seen at $25-50 \%$, but the $r_{\text {exclusion }}$ then reduced below $10 \% \mathrm{BAR}$, although even as little as $1 \%$ gave a measurable bright white area of dye exclusion (Fig. 4e). As expected TEAP silica had no effect on $r_{\text {swell }}$.

\subsection{Imaging dye penetration into capsules}

Imaging experiments were conducted with BAR filled into rings cut from capsules to see if BAR could block dye penetration in the same manner as that observed with tablets. When bromophenol blue solution was added to a capsule segment filled with $25 \%$ cholestyramine with MCC filler, the capsule shell absorbed water for an initial period that varied between experiments, followed by rapid ingress through one or two points on the capsule ring (e.g. Fig. 5a). As the dye solution entered, separation was seen in a similar manner to that seen with tablets, with a dark blue area near the point of ingress, and a bright white area in the rest of the area indicating hydration with dye-depleted water (Fig. 5a). When the extent of dye penetration was measured by drawing around blue and white areas (Fig. 5a), a mean excluded area of around $80 \%$ of the total area was seen (Fig. 5b). When the proportion of cholestyramine was reduced to $4 \%$ however, far less dye exclusion was seen with a mean excluded area less than $50 \%$ of the total area, and more variation between replicate samples was observed (Fig. 5b). When 400 mesh Dowex 1X2 resin was used at 25\% and 50\%, less dye exclusion was seen, and far greater dye exclusion was achieved with the dried disaggregated form. Thus a similar rank of exclusion to that seen with tablets was observed, with cholestyramine best, with dry 400 mesh Dowex 1X2 nearly as good, but aggregated wet resin worst.

\subsection{An optimal formulation for bile protection}

Having established the relative dye exclusion given by different BAR, and that dye could be excluded from capsules as well as tablets, we needed to test if bacteria could be protected from bile solutions in a manner that agreed with the imaging system.

In initial experiments, dissolution of Vcaps ${ }^{\mathrm{TM}}$ HPMC capsules containing dried LBV alone in bile solutions gave an unexpectedly high cell recovery. We therefore tested if filling dried LBV into capsules gave some protection from bile without BAR addition, when compared to powder weighed directly into test tubes without capsules. Surprisingly, a range of different bile toxicity was observed depending on the capsule shell material used (Fig. 6a). With powder alone 623-fold less viable bacteria were recovered in a $1 \%$ bile solution compared to buffer, whereas with gelatin capsules 46-fold less viable bacteria were recovered, representing a protection of 13.5 -fold (Fig. 6a). V caps $^{\mathrm{TM}}$ HPMC capsules gave far higher protection, with only 3.6-fold loss of bacteria or a protection of 174-fold - a 
level of protection comparable to that achieved using $7 \mathrm{~mm}$ diameter $2 \mathrm{~mm}$ thick compressed matrix tablets containing the BAR cholestyramine (Edwards and Slater 2009). Vcaps Plus ${ }^{\mathrm{TM}}$ capsules are made with a modified HPMC shell designed to give far faster release compared to conventional HPMC capsules; with these capsules no significant protection was seen (Fig. 6a). This data demonstrate that some capsules can alone give a degree of protection from bile toxicity for stabilised dry LBV.

In order to test the effect of differing BAR type on bile protection within capsules, it was therefore necessary to use Vcaps Plus ${ }^{\mathrm{TM}}$ HPMC capsules that gave the quickest release but least inherent protection from bile. The imaging studies predicted that protection given by 400 mesh Dowex $1 \mathrm{X} 2$ could be significantly improved simply by removing moisture to generate a free-flowing non-aggregated powder, and indeed when added at $25 \%$ to dried LBV and MCC filler, an impressive difference was seen with dried 400 mesh Dowex 1X2 providing 20-fold protection compared to filler alone in capsules, in contrast to the wet preparation which gave no significant protection (Fig. 6b). Furthermore, again as predicted by the imaging data, cholestyramine gave even better protection than the dried 400 mesh Dowex 1X2, with 27-fold protection compared to filler alone in capsules and 120-fold protection compared to powder without capsules, and showing no significant loss of bacteria in $1 \%$ bile compared to buffer (Fig. 6b).

Finally, we made and tested an optimised formulation in an increased bile concentration. When powder alone without capsules was dispersed in $4 \%$ bile solution, a 4200 -fold loss was observed, in contrast to Vcaps ${ }^{\mathrm{TM}}$ HPMC capsules with filler alone when 40 -fold less bacteria were recovered; this represents a 106-fold protection simply by the capsule shell (Fig. 6c), similar to the 174-fold protection from $1 \%$ bile (Fig. 6a). By adding $25 \%$ cholestyramine $100 \%$ recovery was achieved in $4 \%$ bile, with no difference in cell recovery between bile and buffer (Fig. 6c). This represented a 4200-fold improvement over powder alone without capsules, and a 40-fold improvement over filler in Vcaps ${ }^{\mathrm{TM}}$ HPMC capsules. The composition of pig bile is closer to that of humans (Begley et al. 2005), and a similar high degree of protection from pig bile at $1 \%, 4 \%$ and $8 \%$ was also observed (data not shown), confirming that this optimised formulation can protect dried vaccine bacteria from excess concentrations of bile acids.

To determine the degree of protection provided by the cholestyramine (rather than Vcaps ${ }^{\mathrm{TM}}$ HPMC capsule plus cholestyramine) quick-release Vcaps Plus ${ }^{\mathrm{TM}}$ HPMC capsules were used. Even in these capsules - which gave no protection from $1 \%$ bile (Fig. 6a) - no significant loss of bacteria was detected in $4 \%$ bile compared to buffer when filled with $25 \%$ cholestyramine and dried LBV (Fig. 6c). Therefore simply adding $25 \%$ cholestyramine into dried LBV preparations can provide 4200 -fold protection from $4 \%$ bile.

\section{Discussion}

\subsection{Imaging dye penetration into tablets containing BAR}

The controlled release of drugs adsorbed onto ion exchange resins has been extensively studied, however little is known about penetration of molecules into oral dosages containing ion exchange resins. We describe here how ion exchange resins can also be used to block entry of toxic intestinal molecules into tablets and capsules containing sensitive biopharmaceuticals.

We used the dye bromophenol blue to mimic bile acid behaviour during dissolution of tablets and capsule sections. This dye will not behave identically to bile acids since it differs in size, composition and hydrophobicity. However, it has the same negative charge at $\mathrm{pH} 7$ as bile acids and therefore binds to BAR in a similar manner and it can be simply visualised in real time. Similar images were seen using other anionic dyes, sulforhodamine B and methylene blue (data not shown). The concentration of bromophenol blue used was chosen to give the best contrast when 1-2mm thick tablets were imaged under transmitted light.

As expected, the kinetics of tablet hydration were modulated by compaction force and filler type. In spite of these varying hydration speeds, the appearance of the tablets, in terms of swelling and dye penetration during and after full hydration was very similar regardless of the compaction force or filler. This suggested both swelling and the effect of BAR on dye movement were independent of hydration kinetics. Indeed, when the endpoint $r_{\text {swell }}$ and $r_{\text {exclusion }}$ were plotted against compaction force for both $\mathrm{MCC}$ and starch fillers, they were independent of both torque and the filler used (Fig. 2f, g), and therefore must also by independent of hydration time. Similar values of $r_{\text {swell }}$ and $r_{\text {exclusion }}$ of around $7 \mathrm{~mm}$ 
and around $4.5 \mathrm{~mm}$ respectively were found with total hydration times varying from as little as $15 \mathrm{~s}$ (MCC at 8 N.m; Fig. 2d and f) to as long as 250s (starch at 30 N.m; Fig. 2e and g). Furthermore, far less

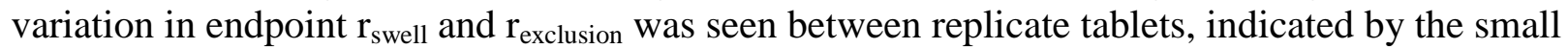
standard deviations seen in these measurements (Fig. 2f, g). This suggested that endpoint $r_{\text {swell }}$ and $\mathrm{r}_{\text {exclusion }}$ values are reproducible measures of dye penetration into tablets.

The purpose of the study was to understand how to gain maximal protection against anion penetration to give optimal defence for dry bacteria from bile acids. The endpoint $r_{\text {exclusion }}$ was chosen as the most important parameter to measure since it indicates the proportion of the tablet that is hydrated without dye (and therefore bile acids). However, this parameter reflects two components: more effective dye binding during hydration will increase the $r_{\text {exclusion}}$, but an increase in $r_{\text {exclusion }}$ will also occur as the tablet matrix swells. Since in many tablets a high degree of swelling was seen, both endpoint $r_{\text {exclusion }}$ and $r_{\text {swell }}$ must be compared in order to fully understand the exclusion properties of any given composition.

The observation that $r_{\text {swell }}$ was independent of filler suggested strongly that the swelling seen was caused predominantly by the cholestyramine; when pure filler tablets of either lactose or calcium phosphate were imaged, swelling was limited to $4 \mathrm{~mm}$ (initial radius $3.5 \mathrm{~mm}$ ), in contrast to tablets containing $50 \%$ cholestyramine which reached $7 \mathrm{~mm}$ (Fig. 3a). The swelling caused by cholestyramine was caused by the polystyrene/DVB backbone of this BAR; similar high swelling was seen with tablets made with a BAR of similar particle size and backbone, polystyrene sulfonate, mixed with MCC filler (Fig. 3a). Thus the high swelling seen is a characteristic of the resin, rather than the anion-exchange capacity or filler. Indeed, different anion-binding additives resulted in two distinct patterns; either highly swelling, with an endpoint $r_{\text {swell }}$ of between 7-9mm and $r_{\text {exclusion }}$ of between 4-5mm (polystyrene and dextran backbones); or little swelling with endpoint $r_{\text {swell }}$ of approximately $4 \mathrm{~mm}$ and $r_{\text {exclusion }}$ between 3$3.5 \mathrm{~mm}$ (cellulose and silica gel backbones or chitosan powder). Because of the difference in swelling between these two groups of BAR, it is difficult to directly compare the relative degree of protection expected from these agents.

Varying the proportion of BAR added allowed a quantitative comparison of different BARs. A comparison of different preparations of Dowex 1X2 showed that smaller particles were more effective at excluding dye, and a wet aggregated preparation was less effective than a dry dispersed sample. For maximal protection, a dis-aggregated dry BAR is required. The 400 mesh Dowex 1X2 resin is hygroscopic and quickly absorbs moisture when stored at atmospheric humidity. This presented two possible problems: firstly, the hydrated resin aggregates and is difficult to mix with other powders; secondly, if a significant mass fraction is water, less resin will be included when weighed into a formulation. Significantly, at $1 \%$ the dried BAR gave higher $r_{\text {exclusion }}$ than $10 \%$ wet sample, indicating that the difference was not simply the increased effective proportion of resin discounting the mass of water included in the wet sample. Instead, the increased dye exclusion may be due to the freely distributed, non-aggregated particle presentation. Although drying improved the dye exclusion at lower proportions, dried 400 mesh Dowex 1 X2 was still far less effective than cholestyramine, confirming the importance of small particle sizes even with equally dry and dispersed BARs (Fig. 4a,c).

It is interesting to consider why a larger particle size and aggregation reduce the ability of BAR to block dye entry, especially since these BAR were supplied with identical chemical composition and capacity for ion binding (table 1). However, the ion binding capacity is measured at equilibrium, and does not reflect the dynamic binding capacity of these resins. A likely explanation is that adsorbents with smaller particle sizes have far higher surface area and thus larger dynamic binding capacity in a situation requiring rapid ion binding, such as the fast ingress of dye solution into a tablet or capsule. To confirm this theory a future study is planned comparing the effect of dynamic binding capacity on dye exclusion.

\subsection{Imaging dye penetration into capsules containing BAR}

Although compressed matrix tablets are the most widely used oral dosage form, they present two major disadvantages for oral live bacterial vaccines. Firstly, compaction can directly kill bacteria and therefore lower compaction forces are required leading to insufficient tablet hardness. Secondly, standard solvents used for enteric coating (either organic solvents or water) can kill or reduce stability of dried live bacteria. In order to remove the requirement for compression, and to separate the live bacteria from coating solvents, hard capsule shells can be used. Crucially, several recent advances now allow 
effective enteric coating of capsules, including sealing procedures and the use of HPMC capsule shells that bind effectively to enteric coating polymers (Huyghebaert et al. 2004).

A comparison of different BARs in capsule rings revealed a similar pattern of dye exclusion to that seen with tablets, although there was more capsule-capsule variation than tablet-tablet variation. Differing amounts of 400 mesh Dowex 1X2 were compared, both in the wet aggregated form and after drying and dispersion. As expected, this BAR was superior when dried, although even after drying, 400 mesh Dowex 1X2 gave less effective dye exclusion area than cholestyramine (Fig. 5b). The increased variation seen with capsules may be due to the variable hydration and broaching of the capsule shell, or from variable powder filling into the capsule rings. In conclusion, imaging dye penetration into capsules showed that BAR can adsorb anions during capsule hydration, and therefore should give protection of bacteria from bile.

\subsection{Validation of imaging results with Live Bacterial Vaccines}

We tested the predictions of the imaging system using bile sensitive room-temperature stable preparations of a model live bacterial vaccine. The protection from bile given by different forms of Dowex 1X2 BAR was as predicted with the imaging studies, with cholestyramine giving the best protection, followed by dried 400 mesh Dowex 1 X2 giving moderate protection, and finally a wet aggregated preparation of 400 mesh Dowex 1 X2 giving no protection (Fig. 6b). As discussed, the likely explanation for superior protection of cholestyramine over the larger particle sizes is that in this dynamic binding situation, the high surface area provided by small particles results in a higher dynamic binding capacity and therefore less bile penetration and toxicity.

The best formulation tested here - $25 \%$ cholestyramine in Vcaps ${ }^{\mathrm{TM}}$ HPMC capsules - gave a higher degree of protection than that seen in a previous study with tablets, and even when tested with very high concentrations of bile effectively complete protection was achieved (Fig. 6c), with 4000-fold more live cell release than powder alone. The inclusion of cholestyramine in the capsules accounted for this high protection, as similar protection was also achieved in Vcaps Plus ${ }^{\mathrm{TM}}$ HPMC capsules

Previous work established that the inclusion of BAR in tablets provides protection from a solution of bile that contains a quantity of bile acids that far exceeds the maximal capacity of the BAR within the tablet (Edwards and Slater 2009). Similarly, in the capsule experiments, the total amount of cholestyramine was $75 \mathrm{mg}$ ( $25 \%$ of $300 \mathrm{mg}$ powder), which can bind a maximum of $218-292 \mu$ mole bile acids (Honda and Nakano 2000). This was added to $25 \mathrm{ml}$ of a $4 \%$ bile solution that contained a total of $2500 \mu$ mole bile acids, representing an 8-11 fold excess of bile acids over BAR capacity, indicating that as with tablets, capsules containing BAR provide temporary protection whilst dry bacteria recover bile tolerance, rather than simply sequestering all bile acids within the test sample.

Surprisingly, simply filling dry LBV into Vcaps ${ }^{\text {TM }}$ HPMC capsules provided a significant degree of protection from bile solutions, whereas Coni Snap ${ }^{\mathrm{TM}}$ gelatin capsules give a lower degree of protection and Vcaps Plus ${ }^{\mathrm{TM}}$ HPMC capsules gave almost no protection (Fig. 6a). This protection can be attributed to retardation of bile acid entry through the hydrated shell into the capsules during dissolution. The mechanism of this protection is likely to be similar to the protection given by BAR: as the capsule shell hydrates it can form a stable gel layer through which water diffuses; in contrast bile acids interact with the shell polymer and are retarded, resulting in reduced bile acid penetration into the bacterial powder. Vcaps ${ }^{\mathrm{TM}}$ HPMC shells will give the most effective protection since they form relatively stable gel layers and bile acids will bind within the gel efficiently via hydrophobic interactions.

Crucially, mixing BAR with the dried bacteria in capsules always gave far greater protection than the capsule alone and the use of BAR for bile protection is a major improvement over the use of protective capsule shells. Furthermore, the increased protection afforded by Vcaps ${ }^{\text {TM }}$ HPMC capsules is tied to their slower release kinetic; less protection is associated with faster releasing capsule shells, and the release kinetic must be tailored to each application depending on the desired location and timing for release of live bacteria. For example, effective vaccination with an LBV may require rapid release of live cells in the upper small intestine and therefore need the quick release properties of Vcaps Plus ${ }^{\mathrm{TM}}$ HPMC capsules.

\section{Conclusions}

We describe here for the first time that transmission imaging can be used to quantitatively determine the effects of inclusion of ion exchange resins on penetration of molecules into tablets and capsules during 
dissolution. This study indicates that anion penetration into both tablets and capsules can be minimised by use of BAR with a smaller particle size, and in a dry, disaggregated form. This suggests that for optimal protection of dried live bacterial vaccine cells showing temporary sensitivity to bile, a BAR with small particle size should be used. The predictions of the imaging study were tested by in vitro determination of live cell release from capsules into bile solutions. The expected protection was observed, with an aggregated wet BAR with large particle size giving no protection; in contrast capsules containing $25 \%$ cholestyramine gave up to 4000 -fold more live cell release into high bile concentrations compared to dried bacteria alone. This is a far higher degree of protection than that previously seen by including cholestyramine in compressed matrix tablets. Furthermore, the use of capsules offers further advantages over tablets by removing the need for compaction that can kill dried bacteria, and by protecting the dried bacteria from water or solvents used in the enteric coating process.

We therefore conclude that an optimised BAR formulation in capsules combined with an enteric coating should be able to efficiently deliver live bacterial vaccines regardless of the bile concentrations encountered on release. Ultimately, the ability of BAR to protect cells from bile and provide a functional formulation for oral vaccines has to be tested in vivo.

Table 1

\begin{tabular}{|c|c|c|c|c|}
\hline & Support & Ligand & $\begin{array}{l}\text { Anion capacity } \\
\text { specified }\end{array}$ & $\begin{array}{l}\text { Measured mean } \\
\text { CE diameter }\end{array}$ \\
\hline Cholestyramine & $\begin{array}{l}\text { Polystyrene-DVB } \\
\text { particles }\end{array}$ & Quaternary Amine, SA & $1 \mathrm{meq} / \mathrm{g}$ & $19.8 \mu \mathrm{m}$ \\
\hline $\begin{array}{l}\text { Dowex 1x2 } 400 \\
\text { mesh }\end{array}$ & $\begin{array}{l}\text { Polystyrene-DVB } \\
\text { beads }\end{array}$ & Quaternary Amine, SA & $3.5 \mathrm{meq} / \mathrm{g}$ & $58 \mu \mathrm{m}$ \\
\hline $\begin{array}{l}\text { Dowex 1x250 } \\
\text { mesh }\end{array}$ & $\begin{array}{l}\text { Polystyrene-DVB } \\
\text { beads }\end{array}$ & Quaternary Amine, SA & $3.5 \mathrm{meq} / \mathrm{g}$ & $297 \mu \mathrm{m}$ \\
\hline QAE sephadex & $\begin{array}{l}\text { Crosslinked } \\
\text { Dextran beads }\end{array}$ & Quaternary Amine, SA & $2.6-3.4 \mathrm{meq} / \mathrm{g}$ & ND \\
\hline TEAE cellulose & Cellulose fibers & Quaternary Amine, SA & $0.75 \mathrm{meq} / \mathrm{g}$ & ND \\
\hline DEAE cellulose & Cellulose fibers & Tertiary Amine, WA & $1 \mathrm{meq} / \mathrm{g}$ & ND \\
\hline TEAP silica & Silica particles & Quaternary Amine, SA & $1.2 \mathrm{mmol} / \mathrm{g}$ & $61 \mu \mathrm{m}$ \\
\hline Chitosan & $\begin{array}{l}\text { Natural } \\
\text { polysaccharide }\end{array}$ & Primary Amine & N/A & ND \\
\hline
\end{tabular}

Table 2

Summary of imaging conditions tested:

\begin{tabular}{|c|c|c|c|c|}
\hline & Compaction torque & Filler & BAR used & $\%$ BAR \\
\hline Figure 2 & 8-30 N.m & MCC vs Starch & Cholestyramine & $50 \%$ \\
\hline \multirow[t]{5}{*}{ Figure $3 \mathrm{a}$} & $8 \mathrm{~N} . \mathrm{m}$ & $\mathrm{MCC}$ & Cholestyramine & $50 \%$ \\
\hline & & Starch & & \\
\hline & & Calcium Phosphate & & \\
\hline & & Silica gel & & \\
\hline & & Lactose & & \\
\hline \multirow[t]{7}{*}{ Figure $3 b$} & $8 \mathrm{~N} . \mathrm{m}$ & $\mathrm{MCC}$ & Cholestyramine & $50 \%$ \\
\hline & & & Dowex $1 \mathrm{X} 2$ & \\
\hline & & & QAE Sephadex & \\
\hline & & & TEAE Cellulose & \\
\hline & & & DEAE Cellulose & \\
\hline & & & TEAP silica & \\
\hline & & & Chitosan & \\
\hline \multirow[t]{3}{*}{ Figure 4} & $8 \mathrm{~N} . \mathrm{m}$ & $\mathrm{MCC}$ & Cholestyramine & $1-50 \%$ \\
\hline & & & Dowex $1 \mathrm{X} 2$ & \\
\hline & & & TEAP silica & \\
\hline
\end{tabular}




\section{Acknowledgements:}

We are grateful to Capsugel for providing information about capsules and capsule technology, and for supplying us with Coni Snap ${ }^{\text {TM }}$ gelatin capsules, Vcaps ${ }^{\text {TM }}$ HPMC capsules and Vcaps Plus ${ }^{\text {TM }}$ HPMC capsules. We thank Rocky Cranenburgh and Matthew Leckenby at Cobra Biomanufacturing for providing the SLDAPD/pUC18I and SL3261/pAH34L model vaccine strains and contributing in discussion of the manuscript. Nuno Reis helped with the imaging studies and discussion of the manuscript. The work was funded by grant project M0024E from the Technology Strategy Board (a business-led executive non-departmental public body, sponsored by the Department for Innovation, Universities and Skills) and the UK Engineering and Physical Sciences Research Council (EPSRC).

\section{References:}

Abramoff, M. D., P. J. Magelhaes, et al., 2004. Image Processing with ImageJ. Biophotonics International 11(7): $36-42$.

Begley, M., C. G. Gahan, et al., 2005. The interaction between bacteria and bile. FEMS Microbiol Rev 29(4): 625-51.

Bergen, S. S., Jr., T. B. Van Itallie, et al., 1959. Effect of an anion exchange resin on serum cholesterol in man. Proc Soc Exp Biol Med 102: 676-9.

Bettini, R., P. Colombo, et al., 1994. Swelling and Drug-Release in Hydrogel Matrices - Polymer Viscosity and Matrix Porosity Effects. European Journal of Pharmaceutical Sciences 2(3): 213-219.

Bullifent, H. L., K. Dhaliwal, et al., 2000. Stabilisation of Salmonella vaccine vectors by the induction of trehalose biosynthesis. Vaccine 19(9-10): 1239-45.

Chirico, S., A. Dalmoro, et al., 2007. Analysis and modeling of swelling and erosion behavior for pure HPMC tablet. Journal of Controlled Release 122(2): 181-188.

Edwards, A. D. and N. K. Slater, 2008. Formulation of a live bacterial vaccine for stable room temperature storage results in loss of acid, bile and bile salt resistance. Vaccine 26(45): 5675-8.

Edwards, A. D. and N. K. Slater, 2009. Protection of live bacteria from bile acid toxicity using bile acid adsorbing resins. Vaccine 27(29): 3897-903.

Garmory, H. S., M. W. Leckenby, et al., 2005. Antibiotic-free plasmid stabilization by operator-repressor titration for vaccine delivery by using live Salmonella enterica Serovar typhimurium. Infect Immun 73(4): 200511.

Honda, Y. and M. Nakano, 2000. Studies on adsorption characteristics of bile acids and methotrexate to a new type of anion-exchange resin, colestimide. Chem Pharm Bull (Tokyo) 48(7): 978-81.

Huyghebaert, N., A. Vermeire, et al., 2004. Alternative method for enteric coating of HPMC capsules resulting in ready-to-use enteric-coated capsules. Eur J Pharm Sci 21(5): 617-23.

Kantele, A., H. Arvilommi, et al., 1991. Comparison of the human immune response to live oral, killed oral or killed parenteral Salmonella typhi TY21A vaccines. Microb Pathog 10(2): 117-26.

Kimoto, H., S. Ohmomo, et al., 2002. Enhancement of bile tolerance in lactococci by Tween 80. J Appl Microbiol 92(1): 41-6.

Levine, M. M., 2006. Enteric infections and the vaccines to counter them: future directions. Vaccine 24(18): 3865-73.

Prakash, S. and M. L. Jones, 2005. Artificial Cell Therapy: New Strategies for the Therapeutic Delivery of Live Bacteria. J Biomed Biotechnol 2005(1): 44-56.

Yoshihashi, Y., M. Makita, et al., 1998. Measurement of rates of water penetration into tablets by microcalorimetry. Chemical \& Pharmaceutical Bulletin 46(3): 473-477. 
Figure 1: Imaging anionic dye penetration into tablets containing BAR. a) Cross sectional representation of the dissolution chamber used to image dye penetration into tablets or capsule sections sandwiched between flexible PDMS sheets held with clear acrylic plates. b) Illustration of dye and water fronts seen during hydration of tablet, and measurements of radii to extent of swelling, dye front and hydration front. c) Illustration of radii measured at endpoint when tablet was fully hydrated.

Figure 2: Effect of changing compaction torque and filler type on hydration kinetics. a-c) Tablets of $50 \%$ cholestyramine and $50 \%$ MCC were compacted at 8 or 30N.m as indicated, and imaged during dissolution in bromophenol blue solution. a) Images taken at indicated times. b, c) Time course of measured radii of swelling, dye exclusion and hydration for the images in a) for tablets compacted with 8 (b) and 30 N.m (c). d-g) Multiple replicate tablets of 50\% cholestyramine and 50\% MCC (d, f) or 50\% starch $(e, g)$ were compacted at differing torques and then imaged during dissolution in bromophenol blue solution. For each tablet video, the total time taken to full hydration, the endpoint radii of swelling, dye exclusion were measured, and mean values of hydration time (d, e) and endpoint radii (f, g) were plotted against compaction torque. Each point is the mean of 4-8 replicate tablet videos; all error bars are shown and indicate 1 standard deviation.

Figure 3: Effect of filler and BAR type on dye exclusion and tablet swelling. a) Multiple tablets of each of the indicated compositions were compacted with 8 N.m torque and imaged during dissolution in bromophenol blue solution, and the endpoint swelling and exclusion radii were measured. b) 50\% MCC was mixed with the indicated agents, multiple tablets were compacted and imaged, and the endpoint swelling and exclusion radii were measured. Each point is the mean of 4-8 replicate tablet videos; all error bars are shown and indicate 1 standard deviation.

Figure 4: Effect of varying the proportion and type of BAR on dye exclusion and tablet swelling. Tablets made with MCC mixed with the indicated proportion of cholestyramine (a), 400 mesh Dowex 1X2 either as supplied (b) or dried (c), 50 mesh Dowex 1X2 (d) or TEAP silica (e) then imaged and the endpoint swelling and exclusion radii were measured. Each point is the mean of 4-8 replicate tablet videos; all error bars are shown and indicate 1 standard deviation.

Figure 5: Imaging dye exclusion by BAR in capsules. Rings cut from the middle of a size $00 \mathrm{Vcaps}^{\mathrm{TM}}$ HPMC capsule were placed on the lower PDMS sheet, filled with the indicated mixtures of BAR and MCC filler, and sandwiched to ensure only the capsule shell contacted the bromophenol blue solution added to the chamber. a) Representative images from a typical video of $25 \%$ cholestyramine, showing penetration through a point in the shell, followed by dye binding and restricted dye entry while the capsule ring contents fully hydrate and appear bright white, indicating hydration with dye-free buffer. The bottom image illustrates the areas identified and measured as Penetrated and Excluded areas. b) Multiple replicate capsule rings were filled with the indicated BAR and filler proportions, imaged, and Penetrated and Excluded areas measured and plotted. Each point is the mean of 3-5 replicate capsule videos; all error bars are shown and indicate 1 standard deviation.

\section{Figure 6: Validation of optimised formulation for protecting a dried live bacterial vaccine from bile}

A model mouse vaccine was dried and mixed with MCC filler, then mixed with BAR or filler and filled into capsules of various shell material as indicated. The indicated powder or capsules were then tested for live cell release in control buffer or bile solutions to test sensitivity to bile toxicity. a) Shows the effect of differing capsule shell material on the toxicity of $1 \%$ bile without BAR added. b) Illustrates the protection from $1 \%$ bile afforded by the indicated BAR types added at $25 \%$ to the Vcaps Plus ${ }^{\mathrm{TM}}$ HPMC capsules. c) A comparison of live cell release in $4 \%$ bile by powder alone, Vcaps ${ }^{\text {TM }}$ HPMC capsules alone, and $25 \%$ cholestyramine in two capsule types. The data shown were obtained using the strain SL3261/pAH34L; similar protection was seen for the vaccine strain SLDAPD/pUC18I. Complete bile protection with $25 \%$ cholestyramine in capsules was seen in 5 independent experiments; the difference in protection between wet vs dry 400 mesh Dowex 1 X2 was seen in 2 experiments; similar protection was seen in Vcaps ${ }^{\mathrm{TM}}$ HPMC capsules vs powder without capsules in 4 experiments. 
Figure 1

Figure 2 